\title{
ANTI-PCNA ANTIBODY AND FACIAL NERVE PALSY
}

Juliana Nery Ferrari (Hospital São Lucas PUCRS, PORTO ALEGRE, RS, Brasil), Eduarda Rovere Grill (Hospital São Lucas PUCRS, PORTO ALEGRE, RS, Brasil), Cássia Elisa Marin (Hospital São Lucas PUCRS, PORTO ALEGRE, RS, Brasil), Douglas Kazutoshi Sato (Hospital São Lucas PUCRS, PORTO ALEGRE, RS, Brasil), Henrique Luiz Staub (Hospital São Lucas PUCRS, PORTO ALEGRE, RS, Brasil)

\section{BACKGROUND}

The proliferating cell nuclear antigen (PCNA) is a rare antinuclear antibody (ANA) pattern (overall prevalence of $0.3 \%$ ). Autoantibodies to PCNA (anti-PCNA) have been strongly linked to the presence of systemic lupus erythematosus (SLE). While common ANA patterns have been extensively studied, the biological role and clinical significance of anti-PCNA are still unknown. There are recent descriptions of anti-PCNA associated with viral hepatitis, myositis and autoimmune thyroiditis. We herein describe a patient with cranial nerve disease palsy and positivity for anti-PCNA.

\section{CASE REPORT}

A 61-year-old female was admitted in hospital due to an incomplete left eyelid closure and facial drooping on the same side starting two days before. She also complained of inability to close the left eye due to burn sensation and tearing of this eye. A recent migratory polyarthralgia was also referred. She had a previous history of diabetes mellitus, hypertension and obesity, without regular medical supervision. On physical examination she presented left peripheral facial palsy and hypoesthesia of the same side. Joint examination was unremarkable. The brain magnetic resonance image was normal. Laboratory studies revealed a positive ANA test (1/80) with a defined PCNA pattern. Rheumatoid factor and anti-Ro antibodies were negative. Viral screen was negative, and thyroid functional was normal. Low levels of vitamin D and vitamin B12 were noted $(17 \mathrm{ng} / \mathrm{ml}$ and $179 \mathrm{pg} / \mathrm{ml}$ respectively). Glycated hemoglobin was 8.4\%. A clinical diagnosis of peripheral facial palsy was confirmed. The patient was treated with intravenous methylprednisolone (1 g per day) for five days followed by oral steroid tapering. The neurological features rapidly improved. The patient was discharged with oral prednisone and vitamin B12 replacement.

\section{CONCLUSION}

Our patient showed a peripheral facial palsy accompanied by polyarthralgia and anti-PCNA positivity. SLE criteria were not fulfilled, and this is an unreported association. Even though the diabetes mellitus and vitamin B12 deficiency might be involved in the cranial nerve palsy, the prompt response to intravenous steroids suggests the hypothesis of autoimmune facial nerve palsy. Further studies may confirm the pathogenic role of anti-PCNA antibodies in peripheral nerve palsies. 